\title{
THE USE OF FUZZY LOGIC WHILE MODELING THE CREDITWORTHINESS OF LEGAL ENTITIES
}

\author{
Olga Palamarchuk ${ }^{1}$
}

\begin{abstract}
The purpose of the article is to develop a methodological approach to support the decision-making process in determining the creditworthiness of legal entities, as well as to create economic mathematical models based on this approach using the theory of fuzzy logic and fuzzy sets. Methodology. In the author's work (Palamarchuk, 2013), 49 real financial statements (Form 1 and Form 2) of Ukrainian enterprises were selected, 25 of which were potentially bankrupt and 24 were normally operating enterprises. As a result, 7 coefficients were obtained. Here we continue our modelling and building rule base. Result of the experiment is based on statistical data of domestic enterprise. The model has been constructed with the use of theory of fuzzy logic. Considering the expert knowledge, this model helps to make decisions on whether to provide the legal entity with the loan. Practical implications. The model and methodology can be used in commercial banks of Ukraine for calculating application risks. The known models do not fit to every economy. This is the reason which provides value originality of the topic of this study, which solves the problem of creating a method that would give the most sufficient assessment of creditworthiness.
\end{abstract}

Key words: creditworthiness, default, fuzzy logic, legal entity, bankruptcy, stable enterprise, term.

JEL Classification: C30, C53, C83, G21, O50

\section{Introduction}

Hundreds of thousands of borrowers apply for loans every day, but not all of them repay them. So, for the bank, who gives loans, it is necessary to build a model that will help to identify the most successful combinations of financial indicators to determine creditworthiness. Moreover, the correct assessment of the creditworthiness of legal entities is a more urgent problem, because the amount of loans they usually take much bigger than for individuals.

Nowadays, to reduce their risks, many banks in Ukraine have given up lending completely. And those who continue to do so use a rather strict framework, beyond which even a fairly promising borrower cannot get the amount he or she needs. Especially, if borrower does not have a credit history.

As for world practice, a significant number of discriminant multifactor models have been developed to create models for assessing the financial condition and creditworthiness of enterprises for different countries, such as Altman for the United States, Taffler and Tisshaw for Great Britain, Beerman for Germany, Davydova and Belikov for Russia, and many others. However, these models are not suitable for Ukrainian enterprises and changing the values of the coefficients does not help. Due to significant differences in the economy of Ukraine and Western countries, it is necessary to take not other values, but other coefficients. The above necessitates the development of a new model for assessing the creditworthiness of legal entities.

\section{Selection of indicators for the model}

The detailed process of selection was described in one of my previous arti-cles (Palamarchuk, 2013). 49 domestic enterprises were selected, 25 of were potentially bankrupt and 24 were normally operating enterprises. As a result, 7 the most informative coefficients were obtained out of 63 ones. On the basis of selected coefficients, a decision will be made whether to grant a loan to a borrower legal entity.

Selected parameters are summarized in Table 1.

\section{Analysis based on the theory of fuzzy logic}

Step 1. Firstly, we select the indicators. We have seven coefficients selected in the work (Palamarchuk, 2013) that are shown in Table 1.

\footnotetext{
Corresponding author:

${ }^{1}$ Kyiv National Economics University named after Vadym Hetman, Ukraine.

E-mail: starosta6101@gmail.com

ORCID: https://orcid.org/0000-0003-0207-5447
} 
Table 1

\begin{tabular}{|c|l|c|c|c|}
\hline Marking & \multicolumn{1}{|c|}{ The name of the coeffi-cient } & $\begin{array}{c}\text { Values indicating } \\
\text { potential bankruptcy }\end{array}$ & Uncertain state & $\begin{array}{c}\text { Values indicat-ing the stable } \\
\text { state of the enterprise }\end{array}$ \\
\hline X1 & Turnover of accounts payable & Less than 0.8 & $0.8-2.2$ & 2.2 and above \\
\hline X2 & Turnover of current assets & Less than 1.5 & - & 1.5 and above \\
\hline X3 & Turnover of equity & Less than 0.6 & $0.6-1.7$ & 1.7 and above \\
\hline X4 & Financial risk & Less than 0.6 & $0.6-1.95$ & 1.95 and above \\
\hline X5 & Concentration of borrowed capital & More than 1.6 & $0.8-1.6$ & 0.5 and below \\
\hline X6 & Debt coverage with equity & Less than 0.18 & $0.18-0.55$ & 0.55 and above \\
\hline X7 & Return on assets & More than 1.2 & - & 1.2 and below \\
\hline
\end{tabular}

Step 2. Now we form linguistic variables. To form a knowledge base while building a model for determining creditworthiness based on fuzzy logic methods, we use three terms for each variable where on the graph in the paper (Palamarchuk, 2013) we obtained two threshold values, and two - for coefficients with one threshold value. Accordingly, a single scale of three qualitative terms is formed: L - low level, M - medium level, $\mathrm{H}$ - high level of the indicator (for two terms the average level is absent).

To estimate the values of the original linguistic variable $\mathrm{Z}$ we will use the terms: $\mathrm{L}$ - do not give credit, $\mathrm{H}$ - give credit.

A term is an any element of a term set. For example, if the system load can be high, medium and low, then «system load» is a linguistic variable; linguistic scores «high», «medium», «low» are terms which together form a term set. The term is described by a fuzzy set using the belonging function. The belonging function is a function that allows to any element of the universal set to determine its degree of belonging to a fuzzy set.

The term L, which characterizes a high degree of risk of bankruptcy and, consequently, low creditworthiness, was decided to assign to those companies whose current ratio is less than the lower limit. Term $\mathrm{H}$ was assigned to those companies for which the value of the coefficient was above the upper limit.

Step 3. Construction of membership functions. Fuzzy descriptions in the structure of the method of financial and economic analysis appear due to the uncertainty of the expert, which arises in the course of various classifications, for example, when the expert cannot clearly distinguish between medium and high levels of a parameter. In this case, it is necessary to construct membership functions of all fuzzy terms of both input and output variables in order to be able to perform an adequate classification of levels of all indicators (Matviichuk, 2007).
For our case, it is convenient to use neither triangular function (which requires three parameters for its task) nor trapezoidal function (which requires four parameters for its task), which are quite popular while using fuzzy logic. We will use the Gauss function. The main advantages of the Gaussian functions are, firstly, simplicity, because they are determined by only two parameters, and secondly, the convenience of setting these parameters, because the functions have fairly simple derivatives. Also, such functions do not fall directly to zero, only asymptotically approaching it, which provides additional advantages during calculation of values of the resulting indicator in models on fuzzy logic.

To construct membership functions, we will use the data in Table 1.

Thus, the values of the main parameters will be set in the form of appropriate terms:

Parameter $\ll \mathrm{X} 1 \gg$ is divided into terms, which are marked as follows: $\mathrm{L}$ - low, $\mathrm{M}$ - medium, $\mathrm{H}$ - high.

Accordingly, the value of the turnover of accounts payable, which shows the rate of turnover of accounts payable for the period under analysis, expansion or decrease of commercial credit provided to the company can be divided into the following terms: $\mathrm{L}-$ low, $\mathrm{H}$ - high.

Step 4. Formation of a set of rules. Before we start building a model based on fuzzy logic in the Matlab environment, we will form the rules due to which the system will work.

For example, an enterprise is characterized by a high risk of bankruptcy and, consequently, low creditworthiness in that case, when the ratios of current assets, turnover of accounts payable, turnover of equity, financial risk and debt coverage with equity are low (can be interpreted by the term $\ll L »)$, while the coefficients of concentration of borrowed capital and return on assets are high (characterized by the term $\ll \mathrm{H} \gg$ ). A similar combination of values of financial ratios, which 


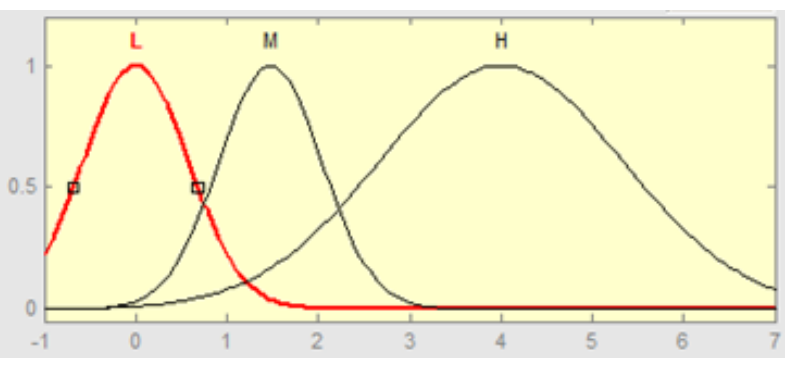

Figure 1. Terms of the linguistic variable $\ll \mathrm{X} 1 \gg-$ the turnover of current assets

characterizes the low creditworthiness of the enterprise $(Z=\langle L \gg)$, is described in the first line of the relevant knowledge base, summarized in Table 2. So, all the set of rules which were deduced by this methodology are presented in the table 2 .

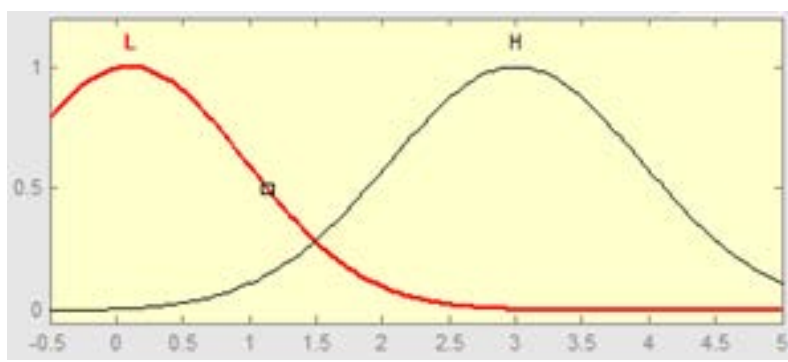

Figure 2. Terms of the linguistic variable $\ll \mathrm{X} 2 »-$ the turnover of accounts payable

Now we will show an analytical form of recording the decision-making rule on the low probability of bankruptcy $\mathrm{H}$ of the company (stable financial condition). For this we will use membership functions and weights:

$$
\begin{aligned}
& \mu^{H}\left(X_{1}, \ldots, X_{7}\right)=w_{1}^{H}\left[\mu^{H}\left(X_{1}\right) \cdot \mu^{H}\left(X_{2}\right) \cdot \mu^{H}\left(X_{3}\right) \cdot \mu^{H}\left(X_{4}\right) \cdot \mu^{L}\left(X_{5}\right) \cdot \mu^{H}\left(X_{6}\right) \cdot \mu^{L}\left(X_{7}\right)\right] \vee \\
& \vee w_{2}^{H}\left[\mu^{H}\left(X_{1}\right) \cdot \mu^{H}\left(X_{3}\right) \cdot \mu^{M}\left(X_{4}\right) \cdot \mu^{L}\left(X_{5}\right) \cdot \mu^{M}\left(X_{6}\right) \cdot \mu^{H}\left(X_{7}\right)\right] \vee \\
& \vee w_{3}^{H}\left[\mu^{M}\left(X_{1}\right) \cdot \mu^{H}\left(X_{2}\right) \cdot \mu^{H}\left(X_{3}\right) \cdot \mu^{H}\left(X_{4}\right) \cdot \mu^{M}\left(X_{5}\right) \cdot \mu^{H}\left(X_{6}\right)\right],
\end{aligned}
$$

where $\mu^{d_{j}}\left(X_{1}, \ldots, X_{N}\right)$ is the membership function of the vector of input variables;

$X_{i}, i=\overline{1, N}$, $\mathrm{j}$-th value of the original variable $\mathrm{Z}$ (linguistic term from the set $d_{j} \in\{L, H\}$ );

$\mathrm{N}$ is the number of input factors (in current task $\mathrm{N}=7$ );

$\mu^{a_{i}^{i p}}\left(X_{i}\right)$ is membership function of the input variable $X_{i}$ of the linguistic term $a_{i}^{j p}, j=\overline{1, m}$, $i=\overline{1, N}, p=\overline{1, k_{j}}\left(\mu^{-a}\left(X_{i}\right)=1-\mu^{a}\left(X_{i}\right)\right)$;

$\mathrm{m}$ is the number of values of the output variable $\mathrm{Z}$ (in current task $=2$ );

$\mathrm{k}_{\mathrm{j}}$ is the number of rules in the knowledge base that are relevant to $j$-th term of the output variable $\mathrm{Z}$.

Since a different number of rules is used to describe each term of the output variable, it is advisable to choose the optimal solution by looking for the rule which has the maximum calculated value of the membership function.
Thus, for the turnover ratio of current assets $\mathrm{X} 1$ (see Figure 1) it was decided to set the distinction between terms $\mathrm{L}$ and $\mathrm{M}$ at the level of 0.8 , and between terms $\mathrm{M}$ and $\mathrm{H}$ at the level of 2.2. For the turnover ratio of accounts payable X2, the boundary between the terms $\mathrm{L}$ and $\mathrm{H}$ was set at 1.5. For the ratios of equity turnover X3 and financial risk $\mathrm{X} 4$, these limits are defined at the level of 0.6 and 1.7 and 0.6 and 1.95, respectively. For the coefficient of concentration of borrowed capital X5 - at the level of 0.8 and 1.6. The debt coverage ratio $\mathrm{X} 6$ as these limits is 0.18 and 0.55 , and the return on assets $\mathrm{X} 7$ is 1.2 .

Step 5. Assessment of the level of indicators. Before assessing the financial condition of the enterprise, the model should be adjusted to the data of bankrupt companies and financially stable enterprises. In order to optimize the model, you

\begin{tabular}{|c|c|c|c|c|c|c|c|c|}
\hline \multicolumn{7}{|c|}{ Linguistic meanings of indicators } & Wage & Output variable \\
\hline$X_{1}$ & $X_{2}$ & $X_{3}$ & $X_{4}$ & $X_{5}$ & $X_{6}$ & $X_{7}$ & $w$ & $Z$ \\
\hline $\mathrm{H}$ & $\mathrm{H}$ & $\mathrm{H}$ & $\mathrm{H}$ & $\mathrm{L}$ & $\mathrm{H}$ & $\mathrm{L}$ & $w_{1}$ & $\mathrm{H}$ \\
\hline $\mathrm{H}$ & none & $\mathrm{H}$ & $\mathrm{M}$ & $\mathrm{L}$ & $\mathrm{M}$ & $\mathrm{H}$ & $w_{2}$ & $\mathrm{H}$ \\
\hline M & $\mathrm{H}$ & $\mathrm{H}$ & $\mathrm{H}$ & M & $\mathrm{H}$ & none & $w_{3}$ & $\mathrm{H}$ \\
\hline $\mathrm{L}$ & $\mathrm{L}$ & $\mathrm{L}$ & $\mathrm{L}$ & $\mathrm{H}$ & $\mathrm{L}$ & $\mathrm{H}$ & $w_{1}^{L}$ & $\mathrm{~L}$ \\
\hline $\mathrm{L}$ & none & $\mathrm{L}$ & M & $\mathrm{H}$ & M & $\mathrm{L}$ & $w_{2}^{L}$ & $\mathrm{~L}$ \\
\hline M & $\mathrm{L}$ & M & $\mathrm{L}$ & $\mathrm{H}$ & $\mathrm{L}$ & none & $w_{3}^{L}$ & $\mathrm{~L}$ \\
\hline
\end{tabular}

Table 2

Knowledge base for determining a company's creditworthiness based on independent variables 
can use the backpropagation algorithm or genetic algorithms (Rothstein, 1999; Rummelhart, Hinton, Williams, 1986). Although, the training of economic mathematical model is not mandatory, because in the presence of basic rules, the model can already issue solutions for any controlled parameters and their values. Nevertheless, if you adjust the parameters of the model to the existing statistical material, the quality of its logical conclusion can be significantly improved.

Data from bankrupt firms and stable companies were used as statistical material. As for the bankrupt enterprises, the analysis of their indicators was carried out at different intervals that remained before the bankruptcy. This was done for the reason various companies show signs of approaching bankruptcy long before the real financial problems. And the sooner we can see the danger and take appropriate action, the better the chances of getting the borrowed money back.

From this point, our model becomes suitable for using to assess the credit-worthiness of the enterprise, although, nevertheless, it is desirable to optimize its parameters on the statistical material, which was done in this work.

Step 6. Decision making. The final solution of the model is chosen in a way that the membership function of the output variable $\mathrm{Z}$ is the largest for the given values of the controlled parameters $\mathrm{X}_{\mathrm{i}}$, $i=\overline{1, N}$ :

$$
Z=\arg \max _{j=1, m}\left[\mu^{d_{j}}\left(X_{1}, \ldots, X_{N}\right)\right],
$$

where $d_{j}=\{L, H\}$.

Since the values of the membership functions of the output variable for each rule are calculated as the product of the membership functions of all input variables, and to determine the term of the performance indicator $\mathrm{Z}$ we use the operation of maximizing the output among all rules, then we will calculate the output variable of the model by next formula:

$$
\mathrm{Z}=\arg \max _{p=1, k_{j}, j=1, \bar{m}}\left\{w_{p}^{d_{j}} \prod_{i=1}^{N} \mu^{a_{i}^{j p}}\left(X_{i}\right)\right\} .
$$

After building the model and setting it up, the model can be used to assess the creditworthiness of enterprise $\mathrm{Z}$ on the basis of indicators $\mathrm{X}_{\mathrm{i}}, i=\overline{1, N}$ to be able to follow the tendency of businesses to loan default.

The result of such a model is not only a linguistic description of the degree of bankruptcy risk, but also the confidence of the expert in the correctness of his or her classification. Thus, the conclusion about the degree of bankruptcy risk of the enterprise acquires not only a linguistic form, but also a description of the quality of the statements.

\section{The result}

After modeling according to our model in the Matlab environment, we obtained the following data: the accuracy of predicting bankruptcies among financially insolvent companies was $89 \%$, the accuracy of classification of financially stable companies was $68 \%$, which was $77.8 \%$ on average for the whole group of analyzed companies.

During the experiments, the system parameters were optimized and the base of the decision rules was specified, as well as the possibility of constructing a fuzzy model based on the selected indicators was confirmed.

As we can see, before the system was set up, almost every second company was considered stable, and after the setup - only 1 bankruptcy out of 25 was considered a stable company, and the program identified 7 stable companies as potential bankruptcies. These results are quite good - only every $25^{\text {th }}$ loan can be repaid. In this case, in addition to the practical application of this model, which takes into account financial indicators, it is worth applying a qualitative assessment of borrowers.

Rules orthogonal to each other were constructed by manual, rough adjust-ment. And fine-tuning is an inverse error propagation algorithm adapted for fuzzy models.

By manual, rough adjustment, orthogonal to each other rules were built. And fine tuning is the algorithm of inverse propagation of an error, which is adapted for fuzzy models.

\section{Conclusions}

The build model has shown a high accuracy of predicting the bankruptcy of domestic enterprises using the developed model, and therefore will help to reject «low-quality» borrowers in the future to minimize the risk of loan default. The optimization of the model parameters on the statistical material was carried out as well.

The model is suitable for usage in commercial banks in order to assess the creditworthiness of the enterprise as a main program and as a useful addition to existing ones. Because usually, commercial banks of Ukraine already have some programs for decision making process regarding lending to legal entities. 


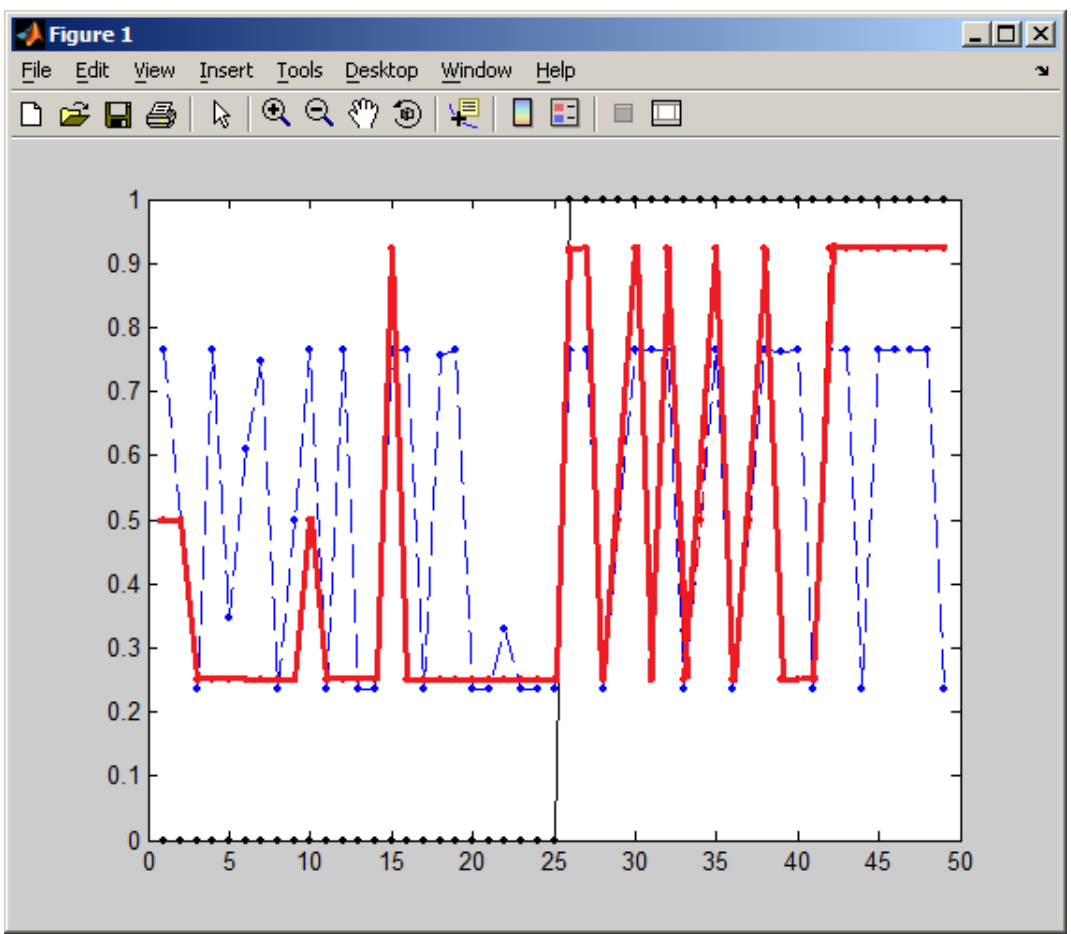

Figure 3. The result in the Matlab environment

(thin line - input data; dotted line - the result before setting up the system; thick line - the result of modeling after using a fuzzy model)

Another advantage of the model is availability. Even small commercial bank can start with analyzing of financial indicators in MS Excel and continue the more deep analysis in the Matlab environment.
But the biggest advantage of the model is, of course, rather big percent of prediction. At the same time it is not big enough, which can lead to retraining of the model.

\section{References:}

Altman, E.I. (1968). Financial Ratios, Discriminant Analysis and the Predic-tion of Corporate Bankruptcy. The Journal of Finance, vol. 4, pp. 589-609.

Davydova, G. V., \& Belikov, A. Yu. (1999). Methods of quantitative assessment of the risk of bankruptcy of enterprises. Risk Management, vol. 3, pp. 13-20.

Matviichuk, A. V. (2007). Diagnosis of bankruptcy of enterprises in the conditions of transformational economy. «Organizational and legal aspects and economic security of modern entrepreneurship»: materials of the IV Regional scientific-practical conference. In 2 parts. Vinnytsia, part I, pp. 80-86.

Matviichuk, A. V. (2006). Discriminant model for estimating the probability of bankruptcy. Modeling and information systems in economics. Kyiv: KNEU, vol. 74, pp. 299-314.

Palamarchuk, O. V. (2013). Selection of indicators to determine the creditworthiness of legal entities. Economic Analysis: Coll. Science. Works. Ternopil, vol. 12(1), pp. 266-268.

Resolution of the Board of the National Bank of Ukraine of January 25, 2012. No. 23.

Resolution of the Board of the National Bank of Ukraine of July 6, 2000. No. 279.

Rothstein, A. P. (1999). Intelligent identification technologies: fuzzy sets, genetic algorithms, neural networks. Vinnytsia: Universum-Vinnytsia.

Rummelhart, D. E., Hinton, G. E., \& Williams, R. J. (1986). Learning Internal Representation by BackPropagation Errors. Nature, vol. 23, pp. 533-536.

Sytnyk, V. F., Tatarchuk, M. I., Pisarevskaya, T. A., \& Sendzyuk, M. A. (2004). Systems of economic information processing: Teaching method. way. for independent study. Kyiv: KNEU.

Tereshchenko, O. O. (2003). Discriminant analysis in assessing the creditworthiness of the enterprise. Bulletin of the NBU, vol. 6(88), pp. 24-27.

Tereshchenko, O. O. (2004). Anti-crisis financial management at the enterprise. Kyiv: KNEU. 